\title{
Front Matter: Volume 10762
}

, "Front Matter: Volume 10762," Proc. SPIE 10762, Hard X-Ray, Gamma-Ray, and Neutron Detector Physics XX, 1076201 (8 November 2018); doi:

$10.1117 / 12.2515799$

SPIE Event: SPIE Optical Engineering + Applications, 2018, San Diego, California, SPIE. United States 


\title{
PROCEEDINGS OF SPIE
}

\section{Hard X-Ray, Gamma-Ray, and Neutron Detector Physics XX}

\author{
Stephen A. Payne \\ Ralph B. James \\ Arnold Burger \\ Michael Fiederle \\ Editors
}

\section{0-21 August 2018}

San Diego, California, United States

Sponsored by

SPIE

Cosponsored by

RMD (United States)

Published by

SPIE 
The papers in this volume were part of the technical conference cited on the cover and title page. Papers were selected and subject to review by the editors and conference program committee. Some conference presentations may not be available for publication. Additional papers and presentation recordings may be available online in the SPIE Digital Library at SPIEDigitalLibrary.org.

The papers reflect the work and thoughts of the authors and are published herein as submitted. The publisher is not responsible for the validity of the information or for any outcomes resulting from reliance thereon.

Please use the following format to cite material from these proceedings:

Author(s), "Title of Paper," in Hard X-Ray, Gamma-Ray, and Neutron Detector Physics XX, edited by Stephen A. Payne, Ralph B. James, Arnold Burger, Michael Fiederle, Proceedings of SPIE Vol. 10762 (SPIE, Bellingham, WA, 2018) Seven-digit Article CID Number.

ISSN: 0277-786X

ISSN: 1996-756X (electronic)

ISBN: 9781510620957

ISBN: 9781510620964 (electronic)

Published by

SPIE

P.O. Box 10, Bellingham, Washington 98227-0010 USA

Telephone +1 3606763290 (Pacific Time) · Fax +1 3606471445

SPIE.org

Copyright (C) 2018, Society of Photo-Optical Instrumentation Engineers.

Copying of material in this book for internal or personal use, or for the internal or personal use of specific clients, beyond the fair use provisions granted by the U.S. Copyright Law is authorized by SPIE subject to payment of copying fees. The Transactional Reporting Service base fee for this volume is $\$ 18.00$ per article (or portion thereof), which should be paid directly to the Copyright Clearance Center (CCC), 222 Rosewood Drive, Danvers, MA 01923. Payment may also be made electronically through CCC Online at copyright.com. Other copying for republication, resale, advertising or promotion, or any form of systematic or multiple reproduction of any material in this book is prohibited except with permission in writing from the publisher. The CCC fee code is 0277$786 \mathrm{X} / 18 / \$ 18.00$.

Printed in the United States of America.

Publication of record for individual papers is online in the SPIE Digital Library.

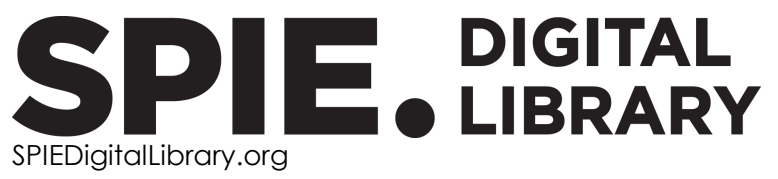

Paper Numbering: Proceedings of SPIE follow an e-First publication model. A unique citation identifier (CID) number is assigned to each article at the time of publication. Utilization of CIDs allows articles to be fully citable as soon as they are published online, and connects the same identifier to all online and print versions of the publication. SPIE uses a seven-digit CID article numbering system structured as follows:

- The first five digits correspond to the SPIE volume number.

- The last two digits indicate publication order within the volume using a Base 36 numbering system employing both numerals and letters. These two-number sets start with $00,01,02,03,04$, 05, 06, 07, 08, 09, 0A, OB ... 0Z, followed by 10-1Z, 20-2Z, etc. The CID Number appears on each page of the manuscript. 


\title{
Contents
}

\author{
$\checkmark \quad$ Authors \\ vii Conference Committee
}

\section{IMAGING DETECTORS}

1076209 Monolithic CMOS detectors for use as x-ray imaging spectrometers [10762-9]

$107620 \mathrm{~A}$ Digital deconvolution of scintillation detector signals for high rate photon-counting $\mathrm{x}$-ray imaging applications [10762-10]

ORGANIC SCINTILLATORS

10762 OB Bismuth-loaded plastic scintillator portal monitors (Invited Paper) [10762-12]

10762 OD Characterization of Polyvinyl Tolvene (PVT) scintillators for fast neutron imaging (Invited Paper) [10762-14]

10762 OF Developments in additive manufacturing of arranged scintillating particle composites for neutron detection [10762-16]

10762 OG Plastic scintillation enhancement through UV emission nanoclusters [10762-17]

PHOTODETECTORS AND APPLICATIONS

$10762 \mathrm{OH} \quad$ Design of low noise silicon reach-through avalanche photodiodes [10762-19]

10762 ol Anticoincidence scintillation detector for the Signal experiment onboard the spacecraft Interhelioprobe [10762-20]

10762 0J Solar neutron and gamma-ray spectrometer for a small satellite [10762-21]

\section{CZT DETECTORS}

10762 ON Development of large-volume high-performance monolithic CZT radiation detector (Invited Paper) [10762-25] 
10762 OR CdZnTe bulk crystal growth and temperature modeling studies at METU-CGL [10762-29]

\section{CZT CONTACTS AND ASICs}

10762 OS Efficient validation testing of Through-Silicon-Via (TSV) ASICs for CZT x-ray detectors [10762-30]

10762 OV Controlling Te inclusion during direct mixed solution growth of large size CdZnTe crystal [10762-35]

TLBR DETECTORS

10762 0X Large area thallium bromide semiconductor radiation detectors with thallium contacts (Invited Paper) [10762-37]

DIAMOND, PEROVSKITE, AND XENON DETECTORS

$107620 Z$ CVD diamond for beta particle detection in a gamma-ray background (Invited Paper) [10762-39]

POSTER SESSION

1076212 Properties of $\mathrm{Cd}_{0.90-\mathrm{x}} \mathrm{Mn}_{\mathrm{x}} \mathrm{Zn}_{0.10 \mathrm{Te}}(\mathrm{x}=\mathbf{0 . 1 0}, \mathbf{0 . 2 0})$ crystals grown by Vertical Bridgman method [10762-46]

1076213 Contrast mediums discrimination by spectral photon-counting CT imaging [10762-47]

1076214 Characterization of Redlen CZT detectors for hard x-ray astronomy [10762-48]

1076218 Pulsed laser deposition of indium on the CdTe crystal surface for contact formation [10762-32] 


\title{
Authors
}

Numbers in the index correspond to the last two digits of the seven-digit citation identifier (CID) article numbering system used in Proceedings of SPIE. The first five digits reflect the volume number. Base 36 numbering is employed for the last two digits and indicates the order of articles within the volume. Numbers start with 00, 01, 02, 03, 04, 05, 06, 07, 08, 09, OA, OB...0Z, followed by 10-1Z, 20-2Z, etc.

\author{
Abbaszadeh, Shiva, ON \\ Allen, Branden, OS \\ Aoki, Toru, 13, 18 \\ Balbaşı, Özden, OR \\ Basak, Arkadip, OS \\ Beck, Patrick R., OB \\ Becla, Piotr, OX \\ Bennett, Paul, $0 Z$ \\ Bolotnikov, A. E., 12 \\ Boyraz, Ozdal, OG \\ Burnham, Jill A., 14 \\ Cao, Lei, OD \\ Chen, Henry, ON \\ Chen, Yongping, $\mathrm{OH}$ \\ Cheng, Zhengxi, $\mathrm{OH}$ \\ Cherepy, Nerine J., OB, OD \\ Chernysheva, Irina $V_{\text {., }}$ 이 \\ Chuirazzi, William, OD \\ Cirignano, Leonard, $\mathrm{OZ}$ \\ Cook, Walter, 14 \\ Datta, Amlan, OX \\ Deng, Z., OA \\ Dmitrenko, Valeriy $V_{\text {., }}$ Ol \\ Doğru, Çiğdem, OR \\ Drury, Owen B., OB \\ Eger, John, ON \\ Ergunt, Yasin, OR \\ Favalli, Andrea, OF \\ Feng, Patrick, OG \\ Fochuk, P., 12 \\ Fujii, Hirosane, 0J \\ Gauron, Thomas, 09 \\ Gnatyuk, Volodymyr, 18 \\ Grachev, Viktor M., Ol \\ Grefenstette, Brian W., 14 \\ Grindlay, Jonathan, OS \\ Harrison, Fiona A., 14 \\ $\mathrm{He}$, Zhong, ON \\ Hehlen, Markus P., OF \\ Hok, Saphon, OB \\ Hong, Jae Sub, OS \\ Hugg, James W., ON \\ Hurlbut, Charles R., OB \\ lanakiev, Kiril D., OF \\ Illiev, Metodi L., OF \\ Inamori, Takaya, OJ \\ Itow, Yoshitaka, OJ \\ James, R. B., 12 \\ Kabukcuoglu, Merve, OR
}

\author{
Kamei, Yuhei, oJ \\ Kanak, A., 12 \\ Kargar, Alireza, $\mathrm{OZ}$ \\ Kenter, Almus, 09 \\ Kim, Hadong, $\mathrm{OZ}$ \\ Kimura, K., 13 \\ Koike, A., 13 \\ Kopach, O., 12 \\ Kopach, V., 12 \\ Kraft, Ralph, 09 \\ Krivova, Kira $V_{\text {., }}$ Ol \\ Li, Handong, ON \\ Li, Mohan, ON \\ Madsen, Kristin K., 14 \\ Martinez, H. Paul, OB, OD \\ Miyasaka, Hiromasa, 14 \\ Miyata, Kikuko, 0J \\ Montemont, Guillaume, ON \\ Motakef, Shariar, OX \\ Nakanishi, Yuta, OJ \\ Nilsson, Mikael, OG \\ Niwa, Takumi, OJ \\ Novikov, Alexander S., 01 \\ Oksuz, Ibrahim, OD \\ Okui, Itsuki, OJ \\ O'Neal, Sean P., OB \\ Parlak, Mehmet, OR \\ Payne, Stephen A., OB \\ Pike, Sean N., 14 \\ Reed, Michael D., ON \\ Sanders, Julian M., 14 \\ Sanner, Robert D., OB \\ Shah, Kanai, $\mathrm{OZ}$ \\ Shcherbak, L., 12 \\ Shustov, Alexander E., Ol \\ Sosanya, Andrew A., 14 \\ Sundaram, Arun G., ON \\ Swanberg, Erik L., $O B$ \\ Tajima, Hiroyasu, OJ \\ Tam, Alan, OG \\ Turan, Rasit, OR \\ Ulin, Sergey E., OI \\ Ünal, Mustafa, OR \\ Uteshev, Ziyaetdin M., Ol \\ Veleschuk, Vitaliy, 18 \\ Verger, Loick, ON \\ Violette, Daniel, OS \\ Vlasik, Konstantin F., Ol \\ Wang, Cheng, OV
}


Wiggins, Brenden W., OF

$\mathrm{Xu}$, Heliang, $\mathrm{OH}$

Yamakawa, Takeharu, 0J

Yamaoka, Kazutaka, OJ

Yue, X. B., OA

Zelenska, Kateryna, 18

Zhang, Hui, OV

Zhang, Song, OV

Zhao, Bo, OV

Zheng, Lili, OV

Zhu, Yuefeng, ON

Proc. of SPIE Vol. 10762 1076201-6

Downloaded From: https://www.spiedigitallibrary.org/conference-proceedings-of-spie on 25 Apr 2023 Terms of Use: https://www.spiedigitallibrary.org/terms-of-use 


\title{
Conference Committee
}

\author{
Program Chairs
}

Ali M. Khounsary, Illinois Institute of Technology (United States)

Ralph B. James, Savannah River National Laboratory (United States)

Conference Chairs

Stephen A. Payne, Lawrence Livermore National Laboratory (United States)

Ralph B. James, Savannah River National Laboratory (United States)

Arnold Burger, Fisk University (United States)

Michael Fiederle, Freiburger Materialforschungszentrum (Germany)

\section{Conference Program Committee}

Toru Aoki, Shizuoka University (Japan)

Gerard Ariño-Estrada, University of California, Davis (United States)

Jim E. Baciak Jr., University of Florida (United States)

Zane W. Bell, Oak Ridge National Laboratory (United States)

Koushik Biswas, Arkansas State University (United States)

Lynn A. Boatner, Oak Ridge National Laboratory (United States)

Aleksey E. Bolotnikov, Brookhaven National Laboratory (United States)

Mary Ellen Braunreuther, Brookhaven National Laboratory (United States)

Giuseppe S. Camarda, Brookhaven National Laboratory (United States)

Bill Cardoso, Creative Electron (United States)

Henry Chen, eV Products, Inc. (United States)

Nerine J. Cherepy, Lawrence Livermore National Laboratory (United States)

Jeffrey J. Derby, University of Minnesota (United States)

Petro M. Fochuk, Yuriy Fedkovych Chernivtsi National University (Ukraine)

Jan Franc, Charles University in Prague (Czech Republic)

Larry Franks, Consultant (United States)

Fei Gao, University of Michigan (United States)

Zhong He, University of Michigan (United States)

Keitaro Hitomi, Tohoku University (Japan)

Alan Janos, U.S. Dept. of Homeland Security (United States)

Mercouri Kanatzidis, Northwestern University (United States)

KiHyun Kim, Korea University (Korea, Republic of) 
Henric Krawczynski, Washington University in St. Louis (United States)

Kelvin G. Lynn, Washington State University (United States)

Krishna C. Mandal, University of South Carolina (United States)

Robert D. McLaren, Consultant (United States)

Shariar Motakef, CapeSym, Inc. (United States)

Sanjoy Mukhopadhyay, International Atomic Energy Agency (United States)

Madan Niraula, Nagoya Institute of Technology (Japan)

Utpal N. Roy, Brookhaven National Laboratory (United States)

Arie Ruzin, Tel Aviv University (Israel)

Narsingh Bahadur Singh, University of Maryland, Baltimore County (United States)

Michael R. Squillante, Radiation Monitoring Devices, Inc. (United States)

Ashley C. Stowe, Y-12 National Security Complex (United States)

Csaba Szeles, Nious Technologies Inc. (United States)

Sergey E. Ulin, National Research Nuclear University MEPhl (Russian Federation)

Edgar V. van Loef, Radiation Monitoring Devices, Inc. (United States)

Aaron L. Washington II, Savannah River National Laboratory (United States)

Richard T. Williams, Wake Forest University (United States)

Ge Yang, North Carolina State University (United States)

Ren-Yuan Zhu, California Institute of Technology (United States)

Session Chairs

1 Inorganic Scintillators

Stephen A. Payne, Lawrence Livermore National Laboratory (United States)

2 Imaging Detectors

Nerine J. Cherepy, Lawrence Livermore National Laboratory (United States)

3 Organic Scintillators

Ren-Yuan Zhu, Caltech (United States)

$4 \quad$ Photodetectors and Applications

Lei Cao, The Ohio State University (United States)

$5 \quad$ CZT Detectors

Robert D. McLaren, Consultant (United States)

6 CZT Contacts and ASICs

Utpal Roy, Brookhaven National Laboratory (United States) 


\section{$7 \quad \mathrm{TIBr}$ Detectors}

Robert D. McLaren, Consultant (United States)

8 Diamond, Perovskite, and Xenon Detectors

Erik L. Swanberg, Lawrence Livermore National Laboratory (United States)

Proc. of SPIE Vol. 10762 1076201-9

Downloaded From: https://www.spiedigitallibrary.org/conference-proceedings-of-spie on 25 Apr 2023 Terms of Use: https://www.spiedigitallibrary.org/terms-of-use 
Proc. of SPIE Vol. 10762 1076201-10

Downloaded From: https://www.spiedigitallibrary.org/conference-proceedings-of-spie on 25 Apr 2023 Terms of Use: https://www.spiedigitallibrary.org/terms-of-use 